\title{
Ion irradiation-induced bimodal surface morphology changes in InSb
}

\author{
Alejandro G Perez-Bergquist ${ }^{1}$, Kundar $\mathrm{Li}^{1}$, Yanwen $\mathrm{Zhang}^{2}$ and \\ Lumin Wang ${ }^{1}$ \\ ${ }^{1}$ Department of Nuclear Engineering and Radiological Sciences, University of Michigan, \\ Ann Arbor, MI 48104, USA \\ ${ }^{2}$ Environmental Molecular Sciences Laboratory, Pacific Northwest National Laboratory, \\ Richland, WA 99352, USA
}

E-mail: lmwang@umich.edu

Received 12 April 2010, in final form 14 June 2010

Published 19 July 2010

Online at stacks.iop.org/Nano/21/325602

\begin{abstract}
High-energy ion irradiation of InSb results in the formation of bimodal surface structures, namely microscale hillock-like structures fully composed of nanoscale fibers. Analysis of the surface structures by a wide range of electron microscopy techniques reveals correlations between the irradiation conditions, such as the ion energy and fluence, and changes in the surface morphology. Sputtering effects play a key role in the integrity of the surface layer with increasing ion fluence. Possible mechanisms responsible for the morphological transformation are discussed, including both irradiation-induced and mechanical effects.
\end{abstract}

\section{Introduction}

Ever since the discovery of semiconducting intermetallic compounds in the 1950s [1], InSb has been widely investigated for use in infrared photovoltaic detectors and photodiodes [2]. Although ion implantation is an attractive way to fabricate junctions in many semiconductor devices, ion irradiation of InSb is known to create extended void defect networks in the material, resulting in the formation of highly porous, fiberlike InSb nanostructures [3-5]. Since the discovery of this phenomenon in InSb, similar ion irradiation-induced porous nanofiber networks have been formed in other semiconductors, including GaSb and Ge [6-9]. Many properties of the porous nanofiber layers, including the fiber layer thickness, the degree of porosity, and the depth of the fiber layer within the sample, can be controlled by varying the ion implantation parameters. Porous nanofiber layers embedded under continuous surface layers have been fabricated both in $\mathrm{InSb}$ and $\mathrm{GaSb}[10,11]$. While porous semiconductor networks have obvious applications in optical lasers and waveguides, they could also have uses as catalyst supports and chemical sensors.

In $\mathrm{GaSb}$, ion irradiation produces uniformly thick nanoporous regions, and embedded nanofibers have flat surface layers [11]. However, in InSb, it has been reported that ion irradiation with $1 \mathrm{MeV} \mathrm{Ga}^{+}$ions produces a slightly buckled surface [10]. In this work, we investigate the change in surface morphology of InSb under heavy ion irradiation as a function of incident ion fluence and energy.

\section{Experimental procedures}

Mirror-polished (100) single crystal InSb wafers with an initial average surface roughness, $R_{a}$, of $<5 \AA$ were sectioned into $1 \mathrm{~cm}^{2}$ samples for ion implantation. The samples were irradiated at room temperature with 1-3 $\mathrm{MeV} \mathrm{Au}^{+}$ions using a National Electrostatics Corporation 9SDH-2 3.0 MV tandem electrostatic accelerator at Pacific Northwest National Laboratories; samples were irradiated to fluences ranging from $5 \times 10^{13}$ ions $\mathrm{cm}^{-2}$ up to a maximum of $8.4 \times 10^{14}$ ions $\mathrm{cm}^{-2}$ ( 3.1-51.4 dpa, or displacements per atom). Additional samples were irradiated with $30 \mathrm{keV} \mathrm{Ga}^{+}$ions in an FEI Nova Nanolab Dualbeam focused ion beam (FIB) system to fluences ranging from $1 \times 10^{15}$ to $1.875 \times 10^{17}$ ions cm $^{-2}$ ( 19-3570 dpa). All samples were stationary and irradiated at a perpendicular incidence angle. Plan-view scanning electron microscopy (SEM) images were taken of the as-irradiated samples using the Dualbeam FIB operated in SEM mode. Samples were then carefully cleaved to avoid any damage to the sample surface. Cross-sectional SEM (XSEM) was performed on the cleaved samples using the FEI Nova SEM with an electron beam incident angle of $90^{\circ}$ to the side of the original sample surface. Crushed powder samples and cross-sectional samples were prepared and observed in a JEOL 


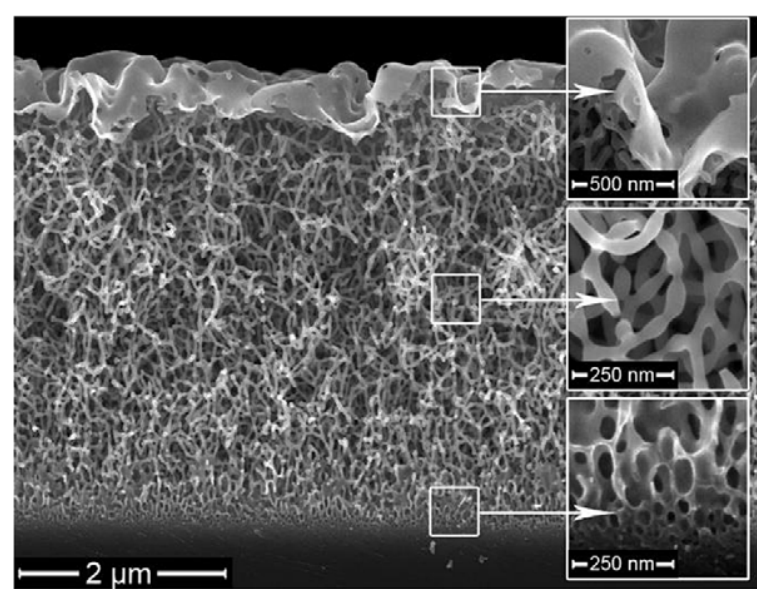

Figure 1. Cross-sectional SEM image of an InSb sample irradiated with $1 \mathrm{MeV} \mathrm{Au}{ }^{+}$to a fluence of $1.25 \times 10^{14}$ ions $\mathrm{cm}^{-2}$ (7.6 dpa). Three distinct regions of the porous layer are visible: a warped yet continuous surface layer, a fiber region composed of fairly uniform InSb fibers, and a formation region composed of small voids embedded within a continuous InSb matrix.

2010F analytical transmission electron microscope (TEM). An EDAX r-TEM detector was used to perform x-ray energy dispersive spectroscopy (XEDS) on the TEM samples. Atomic force microscope (AFM) measurements, which were used in the determination of surface roughnesses, were obtained using a Digital Instruments NanoScope IIIa-Phase AFM. The AFM was operated in tapping mode with an etched single crystal silicon probe. The stopping and range of ions in matter (SRIM) computer code was used to estimate dpa values for each implantation condition [12]. For the SRIM calculations, input values were defined as: a bulk InSb density of $5.77 \mathrm{~g} \mathrm{~cm}^{-3}$, threshold displacement energies of $12.2 \mathrm{eV}$ for In and $16 \mathrm{eV}$ for $\mathrm{Sb}$, and surface binding energies of $2.49 \mathrm{eV}$ for In and $2.72 \mathrm{eV}$ for $\mathrm{Sb}$.

\section{Results and discussion}

Figure 1 shows an XSEM image of an InSb sample irradiated with $1 \mathrm{MeV} \mathrm{Au}{ }^{+}$ions to a fluence of $1.25 \times 10^{14}$ ions cm $^{-2}$
(7.6 dpa). The figure highlights three distinct regions of the porous layer: a highly buckled yet continuous surface layer, a fiber region where the material is composed of fairly uniformly sized and distributed InSb fibers, and a formation region, composed of numerous small voids embedded within a continuous InSb matrix. Figure 2(a) shows a cross-sectional TEM (XTEM) image of a fiber layer irradiated with $2 \mathrm{MeV}$ $\mathrm{Au}^{+}$ions to a fluence of $5 \times 10^{13}$ ions $\mathrm{cm}^{-2}$ (3.1 dpa). XEDS performed on individual nanofibers in the TEM showed that the fibers contained uniform concentrations of In and $\mathrm{Sb}$, and selected area electron diffraction (SAED) of the fiber volume (inset of figure 2(a)) showed that the fibers contain both nanocrystalline and amorphous components. Highresolution TEM (HRTEM) imaging confirmed the presence of randomly oriented nanocrystals in the fibers (figure 2(b)). The primarily polycrystalline nature of the fibers is consistent with results in the literature and indicates that $\mathrm{InSb}$ is amorphized by the incoming ions but that small regions recrystallize due to ion beam heating and thermal spike effects in the material [10]. Similarly, SAED and HRTEM of the surface layer showed that the surface layer was nanocrystalline with some amorphous regions, and XEDS of the surface also confirmed the composition of the surface film as InSb without any significant impurities.

Experimentation across a wide range of ion energies and fluences seems to show that the formation mechanism of InSb fibers is the same as that for GaSb fibers, which has been well established in the literature [7, 11]. Succinctly, ion irradiation creates a large vacancy excess in InSb, leading to the accumulation of vacancies into large voids. These voids accumulate to such a large extent that they force the remaining material in the porous layer into thin nanofibers. As the material accumulates voids and expands, the density of the material drops dramatically, allowing energetic incoming ions to pass through the low-density fiber layer without losing much kinetic energy. This allows for the continual formation of new porous material at the interface of the fiber layer and the dense substrate with further ion irradiation. For example, in InSb bombarded with $1 \mathrm{MeV} \mathrm{Au}^{+}$ions, the sample irradiated to $5 \times 10^{13}$ ions $\mathrm{cm}^{-2}$ formed a porous
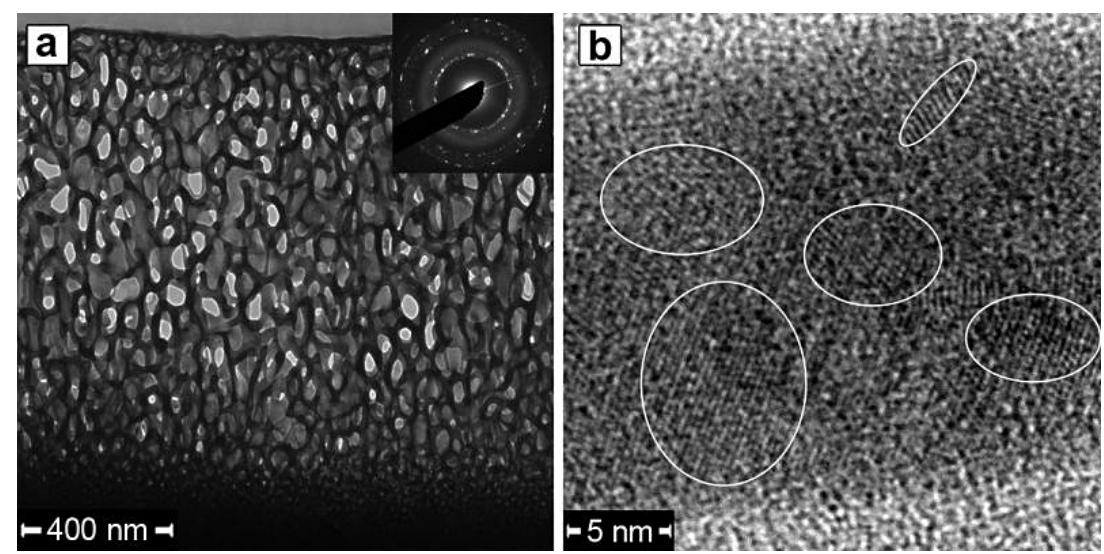

Figure 2. (a) Cross-sectional TEM image of an InSb sample irradiated with $2 \mathrm{MeV} \mathrm{Au}{ }^{+}$to a fluence of $5 \times 10^{13}$ ions $\mathrm{cm}^{-2}(3.1 \mathrm{dpa})$. Inset shows a SAED pattern indicating amorphous and polycrystalline components to the fibers. (b) HRTEM image of an individual fiber, with a number of nanocrystals highlighted. 

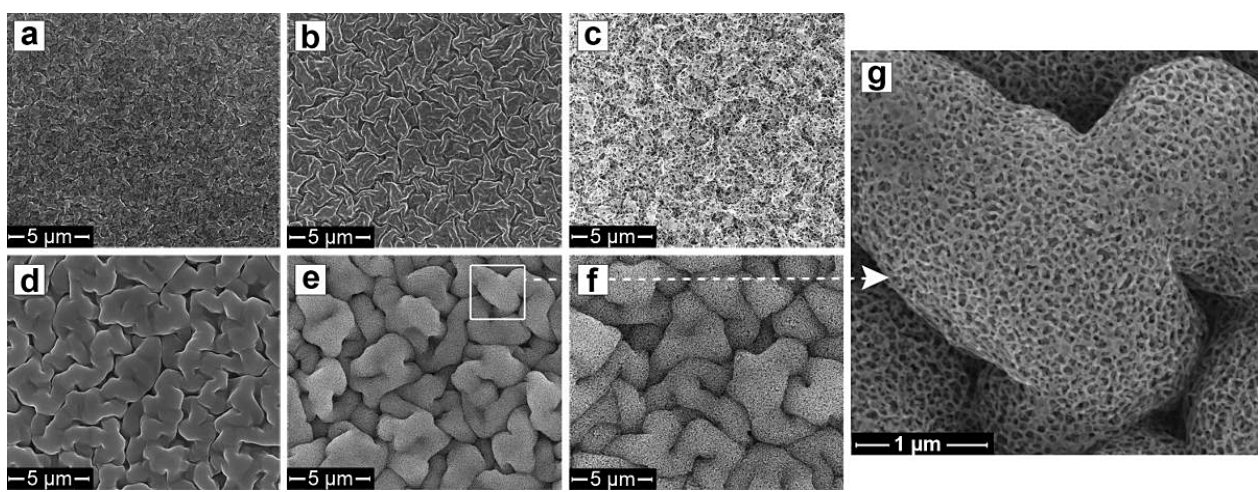

Figure 3. Plan-view SEM images of InSb samples irradiated with 1 and $3 \mathrm{MeV} \mathrm{Au}{ }^{+}$ions. Different samples were irradiated with $1 \mathrm{MeV} \mathrm{Au}^{+}$ to (a) $5 \times 10^{13}$, (b) $1.25 \times 10^{14}$, and (c) $3 \times 10^{14}$ ions cm $\mathrm{cm}^{-2}$ (3.1, 7.6, and $18.4 \mathrm{dpa}$, respectively) and with $3 \mathrm{MeV} \mathrm{Au}^{+}$to (d) $5 \times 10^{13}$, (e) $1 \times 10^{14}$, and (f) $2 \times 10^{14}$ ions cm $\mathrm{cm}^{-2}$ (3.1, 6.1, and $12.2 \mathrm{dpa}$, respectively). In (g) a higher magnification image of (e) is shown. The large surface structures are roughly $2 \mu \mathrm{m}$ across and are composed of nanofibers that are approximately $20 \mathrm{~nm}$ in diameter.

layer roughly $1.8 \mu \mathrm{m}$ in thickness, while at the maximum tested fluence of $8.4 \times 10^{14}$ ions $\mathrm{cm}^{-2}$, a porous layer of $20 \mu \mathrm{m}$ in thickness was produced. Similar fluence effects have previously been reported in the literature [10]. The impact of ion energy is twofold. First, an increase in incident ion energy results in greater energy deposition and greater damage formation. InSb irradiated with $1 \mathrm{MeV} \mathrm{Au}{ }^{+}$ions to $2 \times 10^{14}$ ions $\mathrm{cm}^{-2}$ resulted in formation of a porous layer roughly $6.8 \mu \mathrm{m}$ in thickness, while irradiation of $\mathrm{InSb}$ to the same fluence with $3 \mathrm{MeV} \mathrm{Au}{ }^{+}$ions resulted in a porous layer $18.9 \mu \mathrm{m}$ in thickness. In addition, ion energy influences the depth of peak vacancy production within the irradiated sample, and by irradiating InSb with high-energy ions, the fiber layer forms under a continuous surface layer. Under swift ion irradiation, significant solid surface layers can result; $100 \mathrm{MeV} \mathrm{Sn}^{+}$implantations into InSb have shown porous layer formation beneath a continuous surface layer in excess of $5 \mu \mathrm{m}$ in thickness [13].

In $\mathrm{GaSb}$, ion irradiation with high-energy ions of several hundred kiloelectronvolts to a few megaelectronvolts, depending on the ion species, results in the formation of nanofibers underneath a flat, uniform surface layer. Although embedded fibers can be formed in InSb, the surface layer morphology is textured and varies with both ion energy and ion fluence. Figure 3 shows the surface morphology of InSb samples irradiated with 1 and $3 \mathrm{MeV} \mathrm{Au}{ }^{+}$ions through planview SEM imaging. Irradiation with $1 \mathrm{MeV}$ ions at a fluence of $5 \times 10^{13}$ ions $\mathrm{cm}^{-2}$ (3.1 dpa) leads to surface roughening but no observable pattern formation (figure 3(a)), while increasing the fluence to $1.25 \times 10^{14}$ ions $\mathrm{cm}^{-2}(7.6 \mathrm{dpa}$ ) leads to additional roughening that creates a surface morphology resembling crumpled tissue paper (figure 3(b)). As ion fluence continues to increase, the surface layer is gradually removed, revealing the nanofibers underneath (figure 3(c)). With an increase in ion energy, the surface features became markedly more distinct and noticeable, albeit at lower ion fluence. When irradiated with $3 \mathrm{MeV} \mathrm{Au}^{+}$ions at a fluence of just $5 \times 10^{13}$ ions $\mathrm{cm}^{-2}$ (3.1 dpa), the surface took on a ropy, corrugated appearance (figure 3(d)). An increase in ion fluence caused the surface layer to be gradually removed and caused the features of the

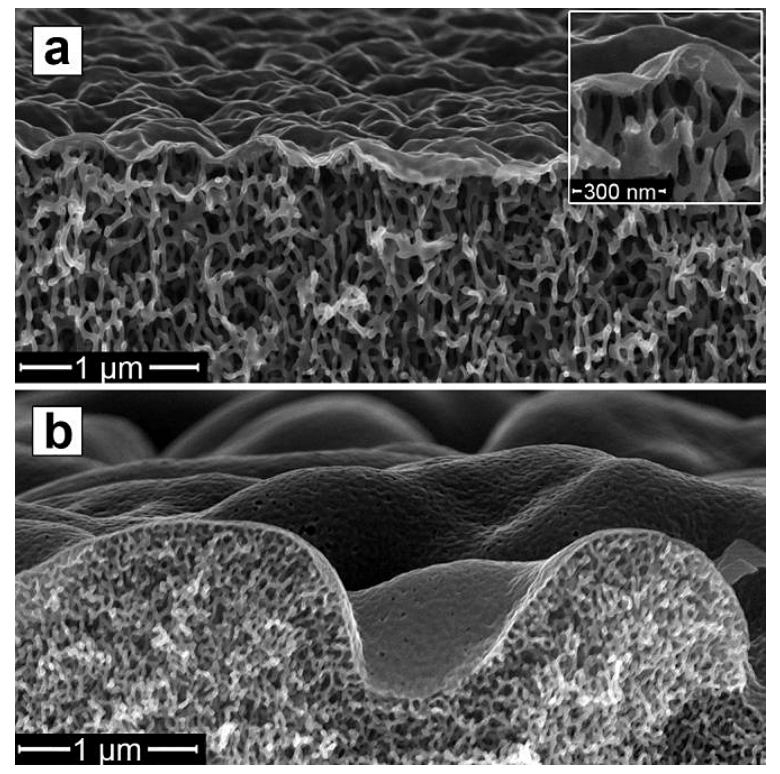

Figure 4. Cross-sectional SEM images of the interface between the surface layer and porous layer in InSb samples irradiated with (a) $1 \mathrm{MeV} \mathrm{Au}^{+}$and (b) $3 \mathrm{MeV} \mathrm{Au}{ }^{+}$ions at $5 \times 10^{13}$ ions cm $\mathrm{cm}^{-2}$ (3.1 dpa). Inset shows a close-up of the attachment of the fibers to the surface. Images were taken at an angle of $10^{\circ}$ from the cross-section surface to better illustrate the surface topography.

ropy regions to become larger but less distinct (figures 3(e) and (f)). The bimodal surface structures that can result from the ion irradiation process are more visible in the higher magnification SEM image shown in figure 3(g), showing that at certain combinations of ion fluence and ion energy the larger microscale hillock-like surface structures are fully composed of individual nanoscale fibers.

When viewed in cross-section, the InSb samples clearly show a thin, continuous surface layer of around $18 \mathrm{~nm}$ in thickness covering a broad expanse of nanofibers. Figure 4 shows SEM cross-sections of the InSb samples, with a focus on the interface between the surface layer and the porous layer, irradiated with 1 and $3 \mathrm{MeV}$ ions at an ion fluence of $5 \times 10^{13}$ ions $\mathrm{cm}^{-2}$. The sample irradiated with $1 \mathrm{MeV}$ ions 
exhibited a maximum peak to valley distance of about $185 \mathrm{~nm}$, where peaks are defined as the highest points of elevation of the microscale hillocks and valleys are defined as the lowest points of elevation between hillocks (figure 4(a)). When the ion energy was increased to $3 \mathrm{MeV}$, the maximum peak to valley distance increased to about $1.2 \mu \mathrm{m}$ (figure $4(\mathrm{~b})$ ).

Overall, increasing the incident ion energy caused an increase in surface structure size and, consequently, a decrease in surface structure areal density. Qualitatively, the effects of incident ion energy on the InSb surface make some sense, as higher energy ions are able to displace more lattice atoms and should be able to create larger defect structures than low energy ions. Quantifying the effects of ion fluence is more difficult due to the narrow range of fluences under which samples with discrete surface layers were observed. Although an increase in ion fluence from $5 \times 10^{13}$ to $1.25 \times 10^{14}$ ions cm $\mathrm{cm}^{-2}$ was observed to cause an increase in surface roughness in the $1 \mathrm{MeV} \mathrm{Au}{ }^{+}$irradiated samples, the most obvious effect of increased fluence across the sample sets was the removal of the surface layer due to atomic sputtering, as seen in figure 3. Due to the fact that both ion energy and fluence directly affect the total energy deposition and dpa of the InSb target, it is difficult to separate the effects of the two parameters. However, the combined total effects of ion damage can be seen by comparing the porous layer thickness, $t$, to the surface roughness, $R_{t}$, of the samples, where:

$$
R_{t}=R_{\mathrm{p}}-R_{\mathrm{v}}
$$

and $R_{\mathrm{p}}$ is the maximum peak height, while $R_{\mathrm{v}}$ is the maximum valley depth. When compared in this fashion, a linear relationship results between $t$ and $R_{t}$, indicating that the evolution of the surface hillocks is a function of the expansion of the porous layer and is not directly related to either ion energy or fluence.

When energetic ions are used to bombard a material, nanoscale surface patterns composed of ripples or dots often result. Most ion-induced surface structures can be explained through the Bradley-Harper $(\mathrm{BH})$ model, which combines Sigmund's sputtering theory with classical surface diffusion arguments to formulate a theory that explains ripple behavior, or through extensions of the basic $\mathrm{BH}$ model [14-18]. The $\mathrm{BH}$ model stipulates that when an ion beam is normally incident on a sample, surface topology differences result in preferential sputtering of low topography regions over high regions, resulting in an instability that leads to the formation of periodic hillocks and depressions. As the $\mathrm{BH}$ model is dependent upon sputtering, its effects are most visible under irradiation conditions where sputtering is dominant, primarily at low ion energies. Figure 5 shows InSb samples irradiated at normal incidence with $30 \mathrm{keV} \mathrm{Ga}{ }^{+}$ions in a dualbeam FIB SEM. The resulting periodic cone-shaped structures present on the irradiated surface seem to represent surface sputtering effects as described by the BH model, although recent work suggests that the specific cone-shaped structures created are a result of combined sputtering and phase separation effects in III-V semiconductors [19]. Nonetheless, the evolution of these structures via low energy ion irradiation indicates that the markedly different surface structures produced via $1-3 \mathrm{MeV}$
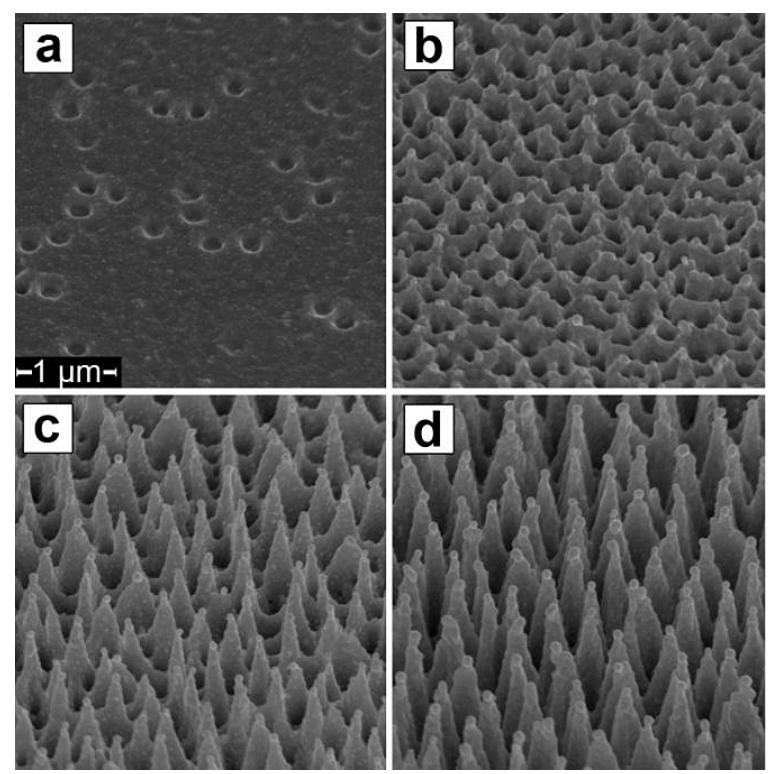

Figure 5. SEM images of InSb irradiated with $30 \mathrm{keV} \mathrm{Ga}^{+}$ions at normal incidence to fluences of (a) $6.25 \times 10^{15}$, (b) $2.5 \times 10^{16}$, (c) $9.375 \times 10^{16}$, and (d) $1.875 \times 10^{17}$ ions $\mathrm{cm}^{-2}(119,476,1786$, and 3570 dpa, respectively). Images are shown at $45^{\circ}$ from normal.

$\mathrm{Au}^{+}$ion irradiation are not a result of sputtering-based effects as described by the $\mathrm{BH}$ model.

For very high-energy ions, surface sputtering and erosion become negligible, but surface modification can still occur due to electronic energy deposition and ionization by incoming ions. Numerous studies have shown that high-energy ion irradiation of amorphous solids results in viscous flow leading to anisotropic growth perpendicular to the ion beam direction, resulting in the expansion of thin films normal to the ion beam and shrinkage parallel to the beam [20-23]. Of course, our case of high-energy ion irradiation of InSb is unique in that volume expansion of the porous layer actually results in a significant expansion of the surface parallel to the ion beam direction. Also, anisotropic growth of the surface layer covering the porous InSb region may be limited due to the relatively lower energy range of the experiments $\left(E \cong 5-15 \mathrm{keV} \mathrm{amu}^{-1}\right)$ as compared to truly high-energy irradiation experiments $(E \geqslant$ $1 \mathrm{MeV} \mathrm{amu}^{-1}$ ).

Although the formation of the porous InSb network is clearly a result of ion irradiation effects, the behavior of the surface layer appears to be a function of mechanical stresses caused by the expansion of the porous layer, as evidenced by the relationship between $t$ and $R_{t}$. Experiments have shown that given the proper set of stress conditions in surfaces, various film morphologies can result, from relatively simple buckled surfaces to labyrinthine wrinkles and bump-like clusters [24-29]. Complex wrinkle structures, in particular, have been observed to form in systems exhibiting a discrete surface film over a compliant substrate that in turn is attached to a rigid support [27]. Such formation is analogous to the irradiation-induced InSb surface, which exhibits a solid InSb film covering a porous fiber network that is in turn attached to a rigid, solid InSb substrate. 

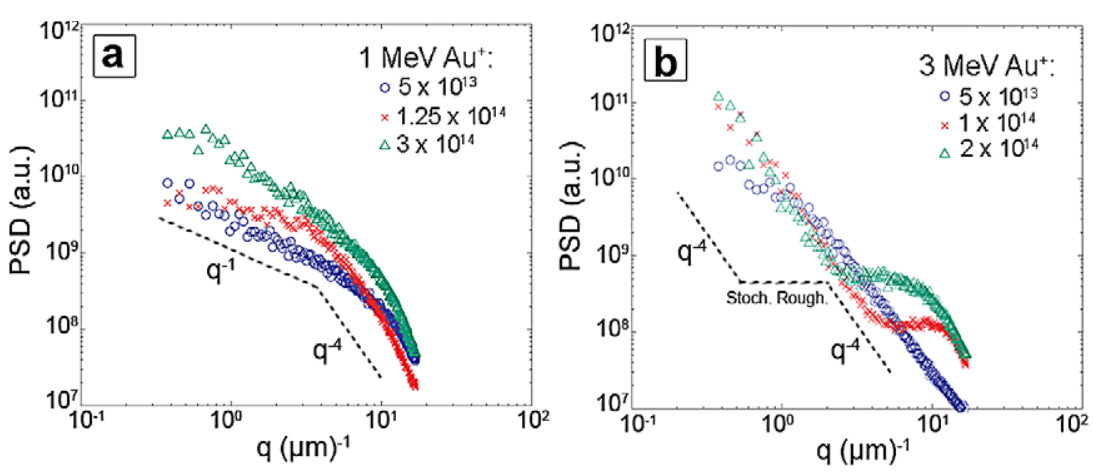

Figure 6. $\mathrm{Log}-\log$ plots of power spectral densities (PSD) for irradiated InSb with (a) $1 \mathrm{MeV} \mathrm{Au}^{+}$and (b) $3 \mathrm{MeV} \mathrm{Au}^{+}$calculated from the images shown in figure 3. Fluences for each ion are listed in units of ions $\mathrm{cm}^{-2}$.

(This figure is in colour only in the electronic version)

Depending on the material composition and crystallinity, surface film thickness, and compliant layer thickness, either tensile or compressive stresses can be generated, leading to varying surface curvatures, roughnesses, and structure sizes [28]. Current theory predicting surface layer behavior is for amorphous systems only, and while TEM evidence showed a large number of nanocrystals in the InSb surface layer, their distribution and orientation indicates that the material is amorphized during ion irradiation. Visual evidence of plastic deformation typical of irradiated amorphous alloys lends credence to this assumption, showing evidence of necking in fibers that attach to the surface film (figure 4(a), inset). In addition, plastic deformation effects have also been seen in high-energy irradiated GaSb in the forms of surface film stretching, delamination, and peeling [11].

Additional insight into relaxation processes in the $\mathrm{InSb}$ system can be obtained by considering the radially-averaged power spectral density (PSD) of the sample surface [28]:

$$
\operatorname{PSD}=\left\langle|h(\vec{q}, t)|^{2}\right\rangle_{|\vec{q}|=q}
$$

where $q$ is the spatial frequency and $h(\vec{q}, t)$ denotes the spatial Fourier transformation of the surface topography $h(\vec{x}, t)$. In the long-time limit, the PSD shows a power-law behavior:

$$
\mathrm{PSD} \propto \frac{1}{\sum_{i=1}^{4} a_{i} q^{i}}
$$

where $a_{i}$ is a constant and exponents for $q^{i}$ are characteristic of atomic surface processes. Based on the shape of the PSD curve, different roughening and smoothening mechanisms can be attributed to the formation processes, including plastic flow, bulk diffusion, surface diffusion, and stochastic and nonstochastic roughening [30]. Figure 6 shows a log-log plot of PSDs, calculated from the InSb surfaces corresponding to figures 3(a)-(f), as a function of spatial frequency. Figure 6 was derived by inputting the individual images of figure 3 into the Matlab computer program, which identifies each image as a grid composed of pixels of varying contrast. The program then completes a fast Fourier transform of each image and outputs the curves shown in figure 6 .

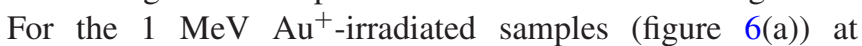

$5 \times 10^{13}$ ions $\mathrm{cm}^{-2}, q^{-1}$ behavior characteristic of viscous flow dominated the relaxation processes, while $q^{-4}$ behavior characteristic of surface diffusion was only observed at higher spatial frequencies. With increasing fluence, the range dominated by $q^{-4}$ spread to lower frequencies, implying that curvature driven surface diffusion processes became more important. For the $3 \mathrm{MeV} \mathrm{Au}^{+}$-irradiated samples (figure 6(b)), $q^{-4}$ behavior dominated relaxation processes in the sample irradiated to $5 \times 10^{13}$ ions $\mathrm{cm}^{-2}$. With an increase of fluence to $1 \times 10^{14}$ ions $\mathrm{cm}^{-2}, q^{-4}$ behavior dominated at low and high frequencies, while at intermediate frequencies, stochastic roughening processes dominated; samples irradiated to $2 \times 10^{14}$ ions $\mathrm{cm}^{-2}$ showed the same fundamental behavior. These increases in surface diffusion at higher ion fluences may help explain the size broadening effects seen in the samples shown in figures 3(d)-(f), while stochastic roughening effects may be tied to sputtering behavior.

\section{Conclusions}

Ion irradiation was used to fabricate layers of InSb nanofibers both with and without continuous surface layers. The surface morphology of the irradiated samples changed as a function of the incident ion energy and fluence, with sputtering effects playing a key role in the integrity of the surface layer at high fluences. The deformation of the surface, while fundamentally tied to irradiation-induced effects such as amorphization and viscous flow of the surface film, seems to primarily be a result of mechanical effects caused by stresses induced during the physical expansion of the porous layer. Further experimental testing and computational modeling are needed to develop a more comprehensive formation model.

\section{Acknowledgments}

This study was supported by the US DOE (DE-FG0202ER46005 and DE-AC05-76RL01830). Y Zhang is supported by the PECASE fund from the Division of Materials Sciences and Engineering of the Office of Basic Energy Sciences, US DOE. A portion of the research was performed using EMSL, a national scientific user 
facility sponsored by the Department of Energy's Office of Biological and Environmental Research and located at Pacific Northwest National Laboratory. Analyses were completed at the University of Michigan's Electron Microbeam Analysis Laboratory using equipment supported in part by the NSF (DMR-0320740 and DMR-9871177).

\section{References}

[1] Weiss H and Welker H 1954 Z. Phys. 138322

[2] Rogalski A 2003 Prog. Quantum Electron. 2759

[3] Destefanis G L and Gailliard J P 1980 Appl. Phys. Lett. 3640

[4] Destefanis G L, Belle J P, Ogier-Collin J M and Gailliard J P 1981 Nucl. Instrum. Methods 182/183 637

[5] Shaanan M, Kalish R and Richter V 1985 Nucl. Instrum. Methods Phys. Res. B 7/8 443

[6] Callec R, Favennec P N, Salvi M, L'Haridon H and Gauneau M 1991 Appl. Phys. Lett. 591872

[7] Nitta N, Taniwaki M, Hayashi Y and Yoshiie T 2002 J. Appl. Phys. 921799

[8] Appleton B R, Holland O W, Narayan J, Schow O E III, Williams J S, Short K T and Lawson E 1982 Appl. Phys. Lett. 41711

[9] Wang L M and Birtcher R C 1989 Appl. Phys. Lett. 552494

[10] Kluth S M, Llewellyn D and Ridgway M C 2006 Nucl. Instrum. Methods Phys. Res. B 242640

[11] Perez-Bergquist A G, Zhu S, Sun K, Xiang X, Zhang Y W and Wang L M 2008 Small 41119

[12] Ziegler J F, Biersack J P and Littmark U 1985 The Stopping and Range of Ions in Solids (New York: Pergamon) Also see: http://www.srim.org
[13] Harris M, Veeramani P, Jayavel P, Hayakawa Y, Kanjilal D and Moorthy Babu S 2006 Nucl. Instrum. Methods Phys. Res. B 244179

[14] Bradley R M and Harper J M E 1988 J. Vac. Sci. Technol. A 62390

[15] Sigmund P 1969 Phys. Rev. 184383

[16] Mullins W W 1957 J. Appl. Phys. 28333

[17] Makeev M A, Cuerno R and Barabasi A L 2002 Nucl. Instrum. Methods Phys. Res. B 197185

[18] Chason E, Mayer T M, Kellerman B K, McIlroy D T and Howard A J 1994 Phys. Rev. Lett. 723040

[19] Le Roy S, Barthel E, Brun N, Lelarge A and Søndergard E 2009 J. Appl. Phys. 106094308

[20] Snoeks E, Polman A and Volkert C A 1994 Appl. Phys. Lett. 652487

[21] Gutzmann A, Klaumünzer S and Meier P 1995 Phys. Rev. Lett. 742256

[22] Trinkaus H and Ryazanov A I 1995 Phys. Rev. Lett. 745072

[23] Gutzmann A and Klaumünzer S 1997 Nucl. Instrum. Methods Phys. Res. B 127/128 12

[24] Matuda N, Baba S and Kinbara A 1981 Thin Solid Films 81301

[25] Iyer S B, Harshavardhan K S and Kumar V 1995 Thin Solid Films 25694

[26] Huang Z, Hong W and Suo Z 2004 Phys. Rev. E 70030601

[27] Huang Z, Hong W and Suo Z 2005 J. Mech. Phys. Solids 532101

[28] Mayr S G and Samwer K 2001 Phys. Rev. Lett. 87036105

[29] Mayr S G, Samwer K and Averback R S 2003 Scr. Mater. 49961

[30] Tong W M and Williams R S 1994 Annu. Rev. Phys. Chem. 45401 